\title{
Efeito do Hidrogel e Ureia na Produção de Mudas de Handroanthus ochraceus (Cham.) Mattos
}

\author{
Cândida Lahís Mews ${ }^{1}$, José Raimundo Luduvico de Sousa ${ }^{1}$, \\ Glauce Taís de Oliveira Sousa Azevedo ${ }^{1}$, Anderson Marcos Souza ${ }^{1}$ \\ ${ }^{1}$ Departamento de Engenharia Florestal, Universidade de Brasília - UNB, Brasília/DF, Brasil
}

\begin{abstract}
RESUMO
Este trabalho avaliou a produção de mudas de Handroanthus ochraceus (Cham.) Mattos submetidas à diferentes doses de hidrogel e adubação nitrogenada em viveiro. $\mathrm{O}$ experimento foi instalado em delineamento inteiramente casualizado, em um fatorial com cinco concentrações de hidrogel $(0$ a 4 g) e seis de adubação ( 0 a $5 \mathrm{~g}$ ), totalizando 30 tratamentos e cinco repetições compostas por quatro mudas. Foram avaliados altura, diâmetro do coleto, número de folhas, massa seca da parte aérea, de raiz e total, relações entre altura/diâmetro do coleto, altura/massa seca da parte aérea, parte aérea/raiz, índice de qualidade de Dickson e incremento em altura e diâmetro em três etapas. Os dados foram submetidos à análise de variância $(p<0,05)$ e regressão. Os diferentes tratamentos geraram respostas distintas e, no geral, influenciaram positivamente o crescimento e a qualidade das mudas. Os 80 dias iniciais sob sombra proporcionaram maior crescimento em altura e diâmetro das mudas.
\end{abstract}

Palavras-chave: ipê-amarelo, viveiro, espécies nativas, recuperação de áreas degradadas.

\section{Effect of Hydrogel and Urea on the Seedling Production of Handroanthus ochraceus (Cham.) Mattos}

\begin{abstract}
This study evaluated the production of seedlings of Handroanthus ochraceus (Cham.) Mattos submitted to different doses of hydrogel and nitrogen fertilization in greenhouse. The experiment was conducted in a completely randomized design in a factorial with five concentrations of hydrogel ( 0 to $4 \mathrm{~g}$ ) and six fertilization treatments ( 0 to $5 \mathrm{~g})$, totaling 30 treatments and five replicates composed of four seedlings. We evaluated height; stem diameter; number of leaves; dry mass of shoot and root; total dry mass; ratios between height/diameter, height/shoot dry mass, and shoot/root; Dickson quality index; and increase in height and diameter in three steps. Data were submitted to analysis of variance $(p<0.05)$ and regression. The different treatments have generated different responses and, in general, positively influenced the growth and quality of seedlings. The initial 80 days under shade provided greater growth in height and diameter of seedlings.
\end{abstract}

Keywords: ipê-amarelo, nursery, native species, recovery of degraded areas. 


\section{INTRODUÇÃO}

O avanço dos problemas ambientais e a necessidade de recuperação de áreas degradadas têm aumentado o interesse sobre o conhecimento das espécies nativas brasileiras (Kageyama \& Gandara, 2000; Melo et al., 2004). Nesse contexto, a necessidade de produção de mudas florestais tem crescido, pois as mudas destinamse tanto a plantios comerciais quanto a programas de restauração ecológica.

Contudo, um dos maiores impasses encontrados nos viveiros e em programas de recomposição vegetal é o alto custo e a falta de domínio das técnicas de produção de mudas e plantios florestais (Sgarbi et al., 1999). Ainda, a utilização de espécies nativas em plantios com fins silviculturais e comerciais torna-se comprometida pelo pouco conhecimento e poucos estudos sobre o comportamento biológico dessas espécies (Bernardi et al., 2012), sobretudo em relação às suas exigências nutricionais e sobre o efeito do hidrogel no crescimento de suas mudas.

Dentre as espécies que apresentam potencial silvicultural encontra-se Handroanthus ochraceus (Cham.) Mattos, que pertence à família Bignoniaceae e é conhecida popularmente como ipê-amarelo. A referida espécie possui hábito arbóreo, distribuição geográfica vasta no Brasil e ocorre principalmente no Cerrado (Lorenzi, 1992; Lohmann, 2012). Handroanthus ochraceus constitui uma espécie que produz anualmente grande quantidade de sementes viáveis e o desenvolvimento das mudas é rápido, ficando prontas para o plantio em campo em menos de cinco meses (Lorenzi, 1992; Silva, 2012). Sua madeira é largamente empregada na construção civil e a planta, em paisagismo regional. Como planta adaptada a terrenos secos, é útil para plantio em áreas degradadas, principalmente em regiões de clima sazonal (Lorenzi, 1992).

Como medida de otimização da produção de mudas de espécies nativas, principalmente para regiões de clima sazonal, caracteriza-se a utilização de hidrogeis incorporados ao substrato. Como produto hidrofílico, o hidrogel absorve e disponibiliza grandes quantidades de água e age como uma reserva para as plantas (Nasser et al., 2007). O resultado é o rápido estabelecimento, redução das perdas por lixiviação de nutrientes, aceleração do desenvolvimento radicular e parte aérea das plantas, bem como redução nos custos com replantio e irrigação (Azevedo et al., 2002). Diversos trabalhos comprovam a eficiência do emprego do hidrogel, principalmente em mudas de espécies florestais de interesse comercial, conforme observado por Martins et al. (2004) (Coffea canephora), Hafle et al. (2008) (Passiflora alata), Azevedo (2000) (Coffea arabica), Saad et al. (2009) (Eucalyptus urograndis), Bernardi et al. (2012) (Corymbia citriodora) e Buzetto et al. (2002) (Eucalyptus urophylla).

Todavia, não há informações técnicas e biológicas sobre a produção de mudas de $H$. ochraceus em função do hidrogel associado à adubação. Buscando fornecer informações que contribuam para a produção de mudas e programas de recomposição florestal, o objetivo do presente estudo foi avaliar o efeito de diferentes doses de hidrogel incorporadas ao substrato e de adubação de cobertura nitrogenada no crescimento de mudas de Handroanthus ochraceus em condições de viveiro.

\section{MATERIAL E MÉTODOS}

O experimento foi conduzido no viveiro da empresa Ecotech Tecnologia Ambiental e Consultoria Ltda, situado em domínio de Cerrado no Distrito Federal ( $16^{\circ} 02^{\prime} 02,57^{\prime \prime}$ S e $\left.47^{\circ} 48^{\prime} 28,42^{\prime \prime} \mathrm{O}\right)$. As sementes de $H$. ochraceus foram semeadas em berços próprios para germinação e as mudas foram repicadas em tubetes de polietileno rígido com capacidade volumétrica de $290 \mathrm{~cm}^{3}$. O substrato utilizado foi composto por uma mistura de solo, esterco bovino, areia e vermiculita (3:1:1:1), calcário dolomítico, macronutrientes (NPK $=4: 30: 16)$ e fertilizante com micronutrientes (Ioorin $=$ $150 \mathrm{~g} / 240 \mathrm{~kg}$ de substrato). A adubação de cobertura foi composta de ureia agrícola ( $\mathrm{N}=46 \%)$ dissolvida em água e aplicada a cada 20 dias após o plantio nos tubetes. As doses foram empregadas conforme os tratamentos $(0,1,2,3,4$ e 5 gramas de ureia por litro de água). O hidrogel utilizado (ForthGel) consistiu em um polímero hidroabsorvente agrícola, o qual foi misturado de forma homogênea ao substrato seco. Após esse processo, os tubetes foram preenchidos com essa mistura. As doses do produto foram aplicadas conforme os tratamentos $(0,1,2,3$ e 4 gramas de hidrogel por litro de substrato). Tais concentrações foram selecionadas de acordo com a recomendação de uso do produto e após experimento piloto em que foi observado que doses maiores que 5 gramas expulsavam parte do substrato do tubete. 
A irrigação das mudas foi efetuada diariamente por aspersão, em quatro frações de 20 minutos. As mudas permaneceram na casa de vegetação durante um período de 80 dias, com nível de sombreamento de $50 \%$. Após esse período, as mudas foram submetidas à rustificação, permanecendo por mais 80 dias a pleno sol.

$\mathrm{O}$ experimento foi implantado em delineamento inteiramente casualizado em esquema fatorial $5 \times 6$, totalizando 30 tratamentos, sendo os fatores as cinco doses de hidrogel e as seis doses de adubação. Em cada tratamento, foram utilizadas cinco repetições compostas de quatro mudas, totalizando 600 mudas.

As características morfológicas das mudas de H. ochraceus foram estudadas por meio da avaliação da altura da parte aérea $(\mathrm{H})$, diâmetro do coleto (DC), número de folhas (NF), massa seca da parte aérea (MSPA), massa seca da raiz (MSR), massa seca total (MST), relação entre a altura da parte aérea e o diâmetro do coleto (RHDC), relação entre a altura da parte aérea e a massa seca da parte aérea (RHMSPA), relação entre a parte aérea e raiz (RPAR) e índice de qualidade de Dickson (IQD). Os valores das etapas de crescimento em altura da parte aérea e diâmetro do coleto foram obtidos a partir dos dados coletados durante o período de desenvolvimento das mudas, aos 80 dias iniciais, após a implantação do experimento, sob sombreamento de $50 \%$, e aos 120 e 160 dias, que caracterizam os 80 dias finais, quando as mudas foram submetidas ao processo de rustificação, a pleno sol.

A determinação da massa seca foi efetuada a partir do material lavado e seco em estufa regulada para
$70{ }^{\circ} \mathrm{C}$ por 72 horas (Gomes et al., 2002). O IQD foi determinado por meio da fórmula IQD $=\mathrm{PMST} /[(\mathrm{HT} /$ DC) + (PMSPA/PMSR)], segundo Dickson et al. (1960).

A normalidade dos dados e a homogeneidade entre variâncias foram verificadas por meio dos testes de Kolmogorov-Smirnov e Levene, respectivamente (Zar, 1999). Para verificar a existência de possíveis diferenças entre os tratamentos foi realizada análise de variância ANOVA e as médias foram comparadas pelo teste de Scott-Knott $(p<0,05)$ (Zar, 1999). Às variáveis que apresentaram diferenças significativas foi aplicada uma análise de regressão a fim de verificar o comportamento das mudas em resposta às diferentes dosagens do polímero associadas às diferentes concentrações de adubação.

\section{RESULTADOS E DISCUSSÃO}

Aos 160 dias, os resultados da análise de variância apontaram diferenças significativas para mudas de Handroanthus ochraceus (Cham.) Mattos nos fatores hidrogel, adubação, bem como na interação entre esses dois fatores (hidrogel*adubação). Os dados mostraram que a aplicação de diferentes dosagens de adubação e hidrogel proporcionaram respostas diferentes das mudas produzidas em condições de viveiro para todas as variáveis testadas, sendo elas H, DC, NF, MSPA, MSR, MST, RHDC, RPAR, RHMSPA e IQD (Tabela 1).

Embora a análise de regressão não tenha indicado diferenças significativas para os fatores hidrogel $\mathrm{e}$ hidrogel*adubação tanto para $\mathrm{H}$ quanto para DC,

Tabela 1. Análise de variância do crescimento e qualidade de mudas de Handroanthus ochraceus (Cham.) Mattos submetidas a diferentes dosagens de hidrogel e adubação em condições de viveiro, em Brasília, DF.

Table 1. Analysis of variance of the growth and quality of seedlings Handroanthus ochraceus (Cham.) Mattos, submitted to different doses of hydrogel and fertilization in greenhouse conditions, Brasília/DF.

\begin{tabular}{|c|c|c|c|c|c|c|c|c|c|c|c|}
\hline \multirow{2}{*}{$\begin{array}{c}\text { Fontes de } \\
\text { variação }\end{array}$} & \multirow[b]{2}{*}{$\mathbf{G L}$} & \multicolumn{10}{|c|}{ Valores do teste $F$} \\
\hline & & $\begin{array}{c}\mathrm{H} \\
(\mathrm{cm})\end{array}$ & $\begin{array}{c}\mathrm{DC} \\
(\mathrm{mm})\end{array}$ & NF & $\begin{array}{c}\text { MSPA } \\
\text { (g) }\end{array}$ & $\begin{array}{c}\text { MSR } \\
(\mathrm{g})\end{array}$ & $\begin{array}{c}\text { MST } \\
(\mathrm{g})\end{array}$ & RHDC & RPAR & $\begin{array}{l}\text { RHM- } \\
\text { SPA }\end{array}$ & IQD \\
\hline Hidrogel & 4 & $28,2^{*}$ & $11,4^{*}$ & $6,0^{*}$ & $16,5^{*}$ & $38,6^{*}$ & $50,4^{*}$ & $5,8^{*}$ & $3,7^{\star}$ & $8,2^{\star}$ & $19,7^{*}$ \\
\hline Adubação & 5 & $330,2^{*}$ & $248,0^{*}$ & $202,6^{*}$ & $82,1^{\star}$ & $44,0^{*}$ & $69,8^{*}$ & $45,9^{*}$ & $21,6^{*}$ & $26,0^{*}$ & $41,3^{*}$ \\
\hline Hidr. ${ }^{\star}$ Adub. & 20 & $26.9^{*}$ & $5,6^{*}$ & $6,9^{*}$ & $4,1^{*}$ & $8,9^{*}$ & $9,4^{*}$ & $18,4^{*}$ & $5,2^{*}$ & $8,4^{*}$ & $14,0^{*}$ \\
\hline Média geral & & 13 & 3,1 & 13,1 & 1,4 & 4,8 & 6,6 & 8,2 & 9,8 & 0,4 & 0,7 \\
\hline CV (\%) & & 29,8 & 10,9 & 17 & 39,1 & 34,1 & 27,8 & 14,4 & 34,7 & 62,7 & 33,3 \\
\hline
\end{tabular}

GL: graus de liberdade; H: altura da parte aérea; DC: diâmetro de coleto; NF: número de folhas; MSPA: massa seca da parte aérea; MSR: massa seca da raiz; MST: massa seca total; RHDC: relação altura/diâmetro do coleto; RPAR: relação parte área/raiz; RHMSPA: relação altura/massa seca da parte aérea; IQD: índice de qualidade de Dickson; CV: coeficiente de variação. *significativo ao nível de 95\% de probabilidade $(p<0,05)$. 
NF e MSPA, a utilização da adubação nitrogenada influenciou de forma positiva o crescimento da parte aérea das mudas. Foi observada tendência à ampliação dos valores em função do aumento das dosagens de adubação até um ponto máximo de crescimento para H (Figura 1a), DC (Figura 1b), NF (Figura 1c) e MSPA (Figura 1d; Figura 1). Para as demais variáveis não houve ajuste dos dados ao modelo de regressão calculado, sendo não significativa a resposta encontrada.

Conforme esperado, a associação de diversos fatores, como as diferentes concentrações de ureia, o tempo de exposição das mudas à fertilização e irrigação podem ter contribuído para melhorar o crescimento das mudas durante o período de permanência em viveiro. Segundo Costa et al. (2006), a adubação, quando agregada a outras características, proporciona condições mais adequadas para o desenvolvimento das plantas, suprindo, essencialmente, suas exigências nutricionais.

Ainda, conforme Sgarbi et al. (1999), o sucesso das adubações, principalmente quando em cobertura, depende basicamente da fonte dos adubos utilizados e das características físicas do substrato e, além disso, de seus aditivos, principalmente hidrogeis (Geesing \& Schmidhalter, 2004). Dessa forma, a fertilização tem sido de fundamental importância na produção de mudas de boa qualidade silvicultural, pois influencia na capacidade de adaptação e crescimento tanto em condições de viveiro como de pós-plantio em campo (Cruz et al., 2006).

$\mathrm{O}$ crescimento máximo das mudas em $\mathrm{H}$ foi atingido com a dose de $3 \mathrm{~g}$ de hidrogel $\mathrm{e} 4 \mathrm{~g}$ de adubação, com média de 20,86 cm, (Figura 2a). Em relação ao DC, a dose que resultou no maior valor médio $(3,11 \mathrm{~mm})$
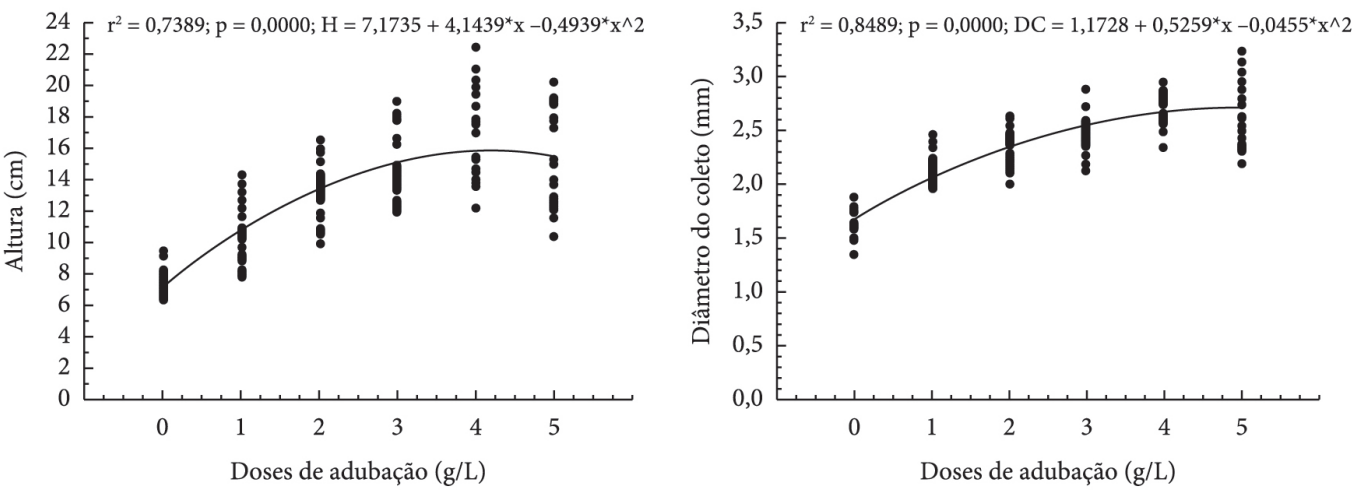

(c)
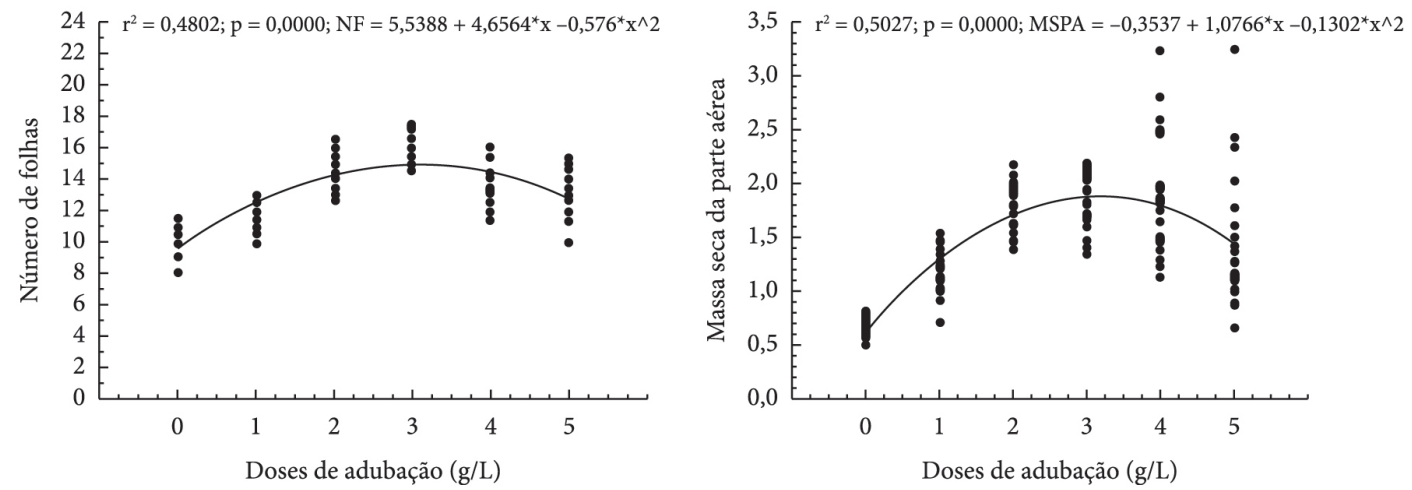

Figura 1. Valores médios de altura da parte aérea (H; a), diâmetro do coleto (DC; b), número de folhas (NF; c) e massa seca da parte aérea (MSPA; d) de mudas de Handroanthus ochraceus (Cham.) Mattos produzidas com diferentes doses de adubação nitrogenada de cobertura em condições de viveiro, em Brasília, DF.

Figure 1. Mean values of shoot height (H; a), stem diameter (DC; b), number of leaves (NF; c) and shoot dry mass (MSPA; d) of seedlings Handroanthus ochraceus (Cham.) Mattos, produced with different nitrogen fertilization doses in greenhouse conditions, Brasília/DF. 
foi de $2 \mathrm{~g}$ de hidrogel associada a $5 \mathrm{~g}$ de adubação (Figura 2b). Para todos os níveis de hidrogel (0 a 4 g), a adubação de $3 \mathrm{~g}$ foi a aplicação que produziu mudas com maior NF, com médias que variaram de 15 a 17 unidades, contudo tais interações não diferiram estatisticamente do tratamento com incorporação de $4 \mathrm{~g}$ de hidrogel e $2 \mathrm{~g}$ de adubação, com média de
15,80 (Figura 2c). Em relação às massas secas, para a MSPA, a concentração de $2 \mathrm{~g}$ de hidrogel e $4 \mathrm{~g}$ de adubação, com 2,66 g de média, foi a dose que se destacou (Figura 2d) e para a MSR (Figura 2e) e MST (Figura 2f), a dosagem que propiciou o maior valor foi de $1 \mathrm{~g}$ de hidrogel e $2 \mathrm{~g}$ de adubação, com média de $5,73 \mathrm{~g}$ e $7,61 \mathrm{~g}$, respectivamente.
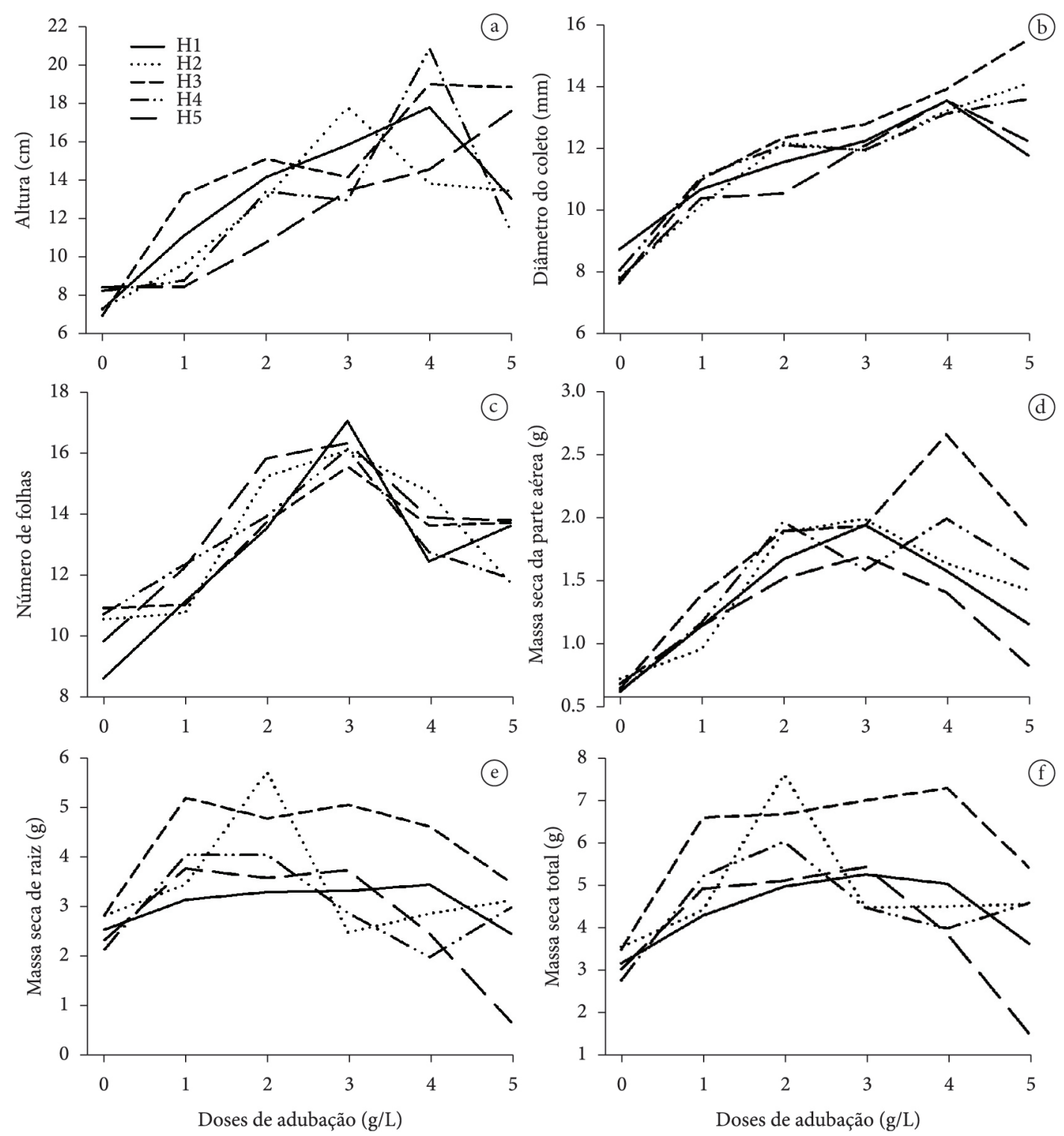

Figura 2. Valores médios de altura (H; a), diâmetro do coleto (DC; b), número de folhas (NF; c), massa seca da parte aérea (MSPA; d), massa seca da raiz (MSR; e), massa seca total (MST; f) para mudas de Handroanthus ochraceus (Cham.) Mattos submetidas às diferentes doses de adubação e hidrogel em condições de viveiro, em Brasília, DF; H: hidrogel.

Figure 2. Mean values of height (H; a), stem diameter (DC; b), number of leaves (NF; c), shoot dry mass (MSPA; d), root dry mass (MSR; e), total dry mass (MST, f) for seedlings Handroanthus ochraceus (Cham.) Mattos, submitted to different doses of fertilization and hydrogel in greenhouse conditions. H: hydrogel, Brasília/DF. 
Resultados eficazes em relação ao emprego do hidrogel e adubação também foram verificados por Bernardi et al. (2012), que observaram que a incorporação de hidrogel e fertilização proporcionaram efeitos positivos no crescimento de mudas de Corymbia citriodora ((Hook.) K. D. Hill e L. A. S. Johnson). Os referidos autores observaram maior incremento em altura $(22,99 \%)$ e diâmetro $(23,12 \%)$ para mudas produzidas com $6 \mathrm{~g}$ do gel por litro de substrato, quando comparadas com mudas produzidas sem a adição do produto, na mesma adubação. Quanto ao uso e eficácia da adubação, Lopes et al. (1999) verificaram em que a aplicação de ureia em cobertura influenciou positivamente o crescimento da parte aérea e das raízes das mudas de maracujazeiro até a dose $0,908 \%$. Uma nutrição nitrogenada adequada automaticamente melhora os teores foliares de nitrogênio $(\mathrm{N})$ e fósforo (P), aumentando, consequentemente, o crescimento e a produção de mudas de qualidade.
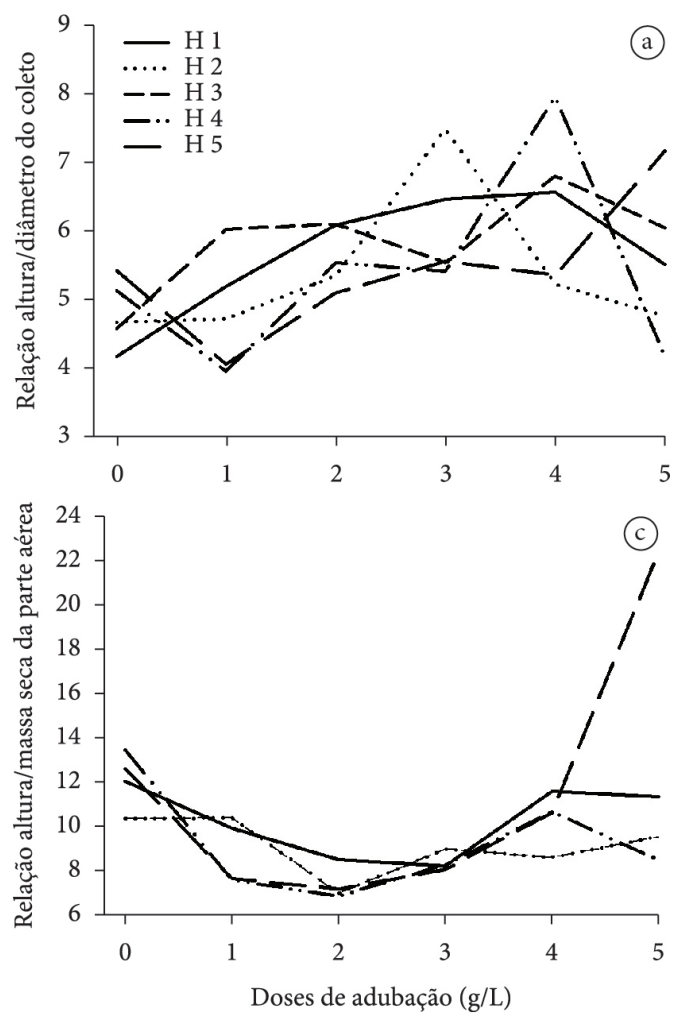

Em relação aos índices de qualidade de mudas, para a RHDC (Figura 3a), a maior média $(7,96)$ foi verificada quando se aplicaram as doses de $3 \mathrm{~g}$ de hidrogel/ litro de substrato e $4 \mathrm{~g}$ de ureia/litro de água. Para as relações RPAR (Figura 3b) e RHMSPA (Figura 3c), a interação que apresentou maior valor médio, 1,39 e 22,35 , respectivamente, foi $4 \mathrm{~g}$ de hidrogel associadas a $5 \mathrm{~g}$ de adubação e, para o IQD (Figura 3d), o tratamento que apresentou maior média $(1,34)$ foi observado nas concentrações de $1 \mathrm{~g}$ de hidrogel e $2 \mathrm{~g}$ de adubação.

Segundo Carneiro (1995), o padrão de qualidade de mudas varia entre as espécies, sendo que o objetivo é alcançar qualidade em que as mudas apresentem capacidade de se desenvolverem em viveiro e oferecer resistência às condições adversas após o plantio. Em estudo realizado por Sousa et al. (2013) foi verificado que o emprego de $6 \mathrm{~g}$ de hidrogel no substrato proporcionou resultados satisfatórios para a razão altura/diâmetro do coleto em mudas de Anadenanthera peregrina (L.)

Figura 3. Valores médios da relação altura/diâmetro do coleto (a), relação parte aérea/raiz (b), relação altura/massa seca da parte aérea (c) e índice de qualidade de Dickson (d) para mudas de Handroanthus ochraceus (Cham.) Mattos submetidas a diferentes doses de adubação e hidrogel em condições de viveiro em Brasília, DF; H: hidrogel.

Figure 3. Mean values of height/stem diameter ratio (a), shoot/root ratio (b), heigh/ shoot dry mass ratio (c) and Dickson quality index (d) for seedlings Handroanthus ochraceus (Cham.) Mattos, submitted to different doses of fertilization and hydrogel in greenhouse conditions. H: hydrogel, Brasília/DF. 
SPEG., mostrando que, assim como no presente estudo, o uso do gel têm-se mostrado eficiente na produção de mudas das referidas espécies. Em relação à produção de folhas, efeitos com a adição de adubação também foram encontrados por Lima et al. (2001) que, estudando o crescimento de mudas de cajueiro anão precoce CCP-76, verificaram que as plantas que receberam fertilizante mineral com ureia responderam satisfatoriamente à adubação e tiveram valores que oscilaram entre 6,0 e 13,66 unidades para as doses $3(6,18 \mathrm{~g})$ e $2(3,34 \mathrm{~g})$ de adubação, respectivamente.

De acordo com a Tabela 2, para o Incremento 1, a H e o DC apresentaram respostas distintas em relação às diferentes doses de hidrogel, adubação, bem como à interação entre esses dois fatores (hidrogel*adubação). Para o Incremento 2, a $\mathrm{H}$ foi significativa para os três fatores avaliados e o DC apresentou respostas distintas somente para o fator adubação, o que indica que não houve efeito do hidrogel e hidrogel*adubação no incremento em diâmetro. Já para o Incremento 3, a $\mathrm{H}$ foi divergente para os três fatores testados, contudo o DC se distinguiu entre as doses somente para os fatores adubação e hidrogel*adubação, indicando que o hidrogel não estava exercendo influência no incremento das mudas.

Pôde-se observar, conforme a Figura 4, que o Incremento 1, que corresponde aos 80 dias iniciais após a implantação do experimento, proporcionou as maiores médias tanto para $\mathrm{H}$ (Figura 4A), como para DC (Figura 4B). Para a $\mathrm{H}$, no Incremento 1 , o teste de média indicou a interação de $2 \mathrm{~g}$ de hidrogel associada a $5 \mathrm{~g}$ de adubação como a melhor média $(8,68 \mathrm{~cm})$, para o Incremento 2 , a associação de $0 \mathrm{~g}$ de hidrogel com 3 g de adubação $(5,50 \mathrm{~cm})$ se destacou em relação às demais e, para o Incremento 3, a dose de 3 de hidrogel em conjunto com a adubação de $4 \mathrm{~g}$ $(2,68 \mathrm{~cm})$ - essas foram as melhores interações para o crescimento em H. Em relação ao DC, para o Incremento 1 , a incorporação de $3 \mathrm{~g}$ de hidrogel no substrato com $2 \mathrm{~g}$ de adubação apresentou superioridade, com média de 1,22 mm e, para o Incremento 3, o maior valor médio foi observado na adubação de $3 \mathrm{~g}$ associada as doses de 2 e $3 \mathrm{~g}$ de hidrogel, com valores de 0,49 e $0,48 \mathrm{~mm}$, respectivamente. Para o Incremento 2 , a análise de variância não mostrou diferenças significativas. Considerando-se esses resultados, nota-se que as duas variáveis avaliadas, $\mathrm{H}$ e DC, são relevantes para se estudar o incremento inicial das mudas de $H$. ochraceus e que as dosagens que mais se destacaram em valores de incremento se concentraram entre 2 a $4 \mathrm{~g}$, tanto para ureia como para hidrogel.

O menor incremento apresentado pelas mudas quando expostas a pleno sol pode ser explicado pelo choque causado pelas diferenças de temperatura, intensidade de luz e umidade de quando expostas a sombreamento. Ferrari \& Shimizu (2005) citam que o fato de rustificar as mudas as leva a manifestar efeitos inibitórios de crescimento resultantes de alterações fisiológicas (diminuição do potencial hídrico foliar, ajuste osmótico, acúmulo de ácido abscísico) e morfológicas (redução da área foliar e aumento da lignificação do caule). Além disso, um grau inadequado

Tabela 2. Análise de variância dos incrementos em altura da parte aérea (H) e diâmetro do coleto (DC) de mudas de Handroanthus ochraceus (Cham.) Mattos submetidas a diferentes dosagens de hidrogel e adubação produzidas em condições de viveiro em Brasília, DF.

Table 2. Analysis of variance of the increments in shoot height (H) and stem diameter (DC) seedlings Handroanthus ochraceus (Cham.) Mattos, submitted to different doses of hydrogel and fertilization produced in greenhouse conditions, Brasília/DF.

\begin{tabular}{|c|c|c|c|c|c|c|c|}
\hline \multirow{3}{*}{ Fontes de variação } & \multirow{3}{*}{ GL } & INCR 1 & -40 dias) & \multicolumn{2}{|c|}{ INCR 2 (120-80 dias) } & \multicolumn{2}{|c|}{ INCR 3 (160-120 dias) } \\
\hline & & \multicolumn{6}{|c|}{ Valores do teste $F$} \\
\hline & & $\mathrm{H}(\mathrm{cm})$ & $\mathrm{DC}(\mathrm{mm})$ & $\mathrm{H}(\mathrm{cm})$ & $\mathrm{DC}(\mathrm{mm})$ & $\mathrm{H}(\mathrm{cm})$ & $\mathrm{DC}(\mathrm{mm})$ \\
\hline Hidrogel & 4 & $62,10^{*}$ & $9,55^{\star}$ & $34,66^{*}$ & $1,22 \mathrm{~ns}$ & $2,05^{\star}$ & $1,00 \mathrm{~ns}$ \\
\hline Adubação & 5 & $294,85^{*}$ & $19,86^{*}$ & $253,57^{\star}$ & $108,65^{*}$ & $57,49^{*}$ & $25,49^{*}$ \\
\hline Hidrogel*adubação & 20 & $21,32^{*}$ & $4,45^{*}$ & $12,42^{*}$ & $0,83 \mathrm{~ns}$ & $7,55^{\star}$ & $2,29^{*}$ \\
\hline Média geral & & 4,81 & 0,89 & 2,37 & 0,26 & 1,45 & 0,22 \\
\hline CV $(\%)$ & & 8,84 & 12,58 & 16,99 & 51,4 & 23,19 & 52,47 \\
\hline
\end{tabular}

GL: graus de liberdade; INCR: incremento; CV: coeficiente de variação. ${ }^{*}$ significativo ao nível de $5 \%$ de probabilidade; ns: não significativo $(p<0,05)$. 
de rustificação pode levar a muda à dormência, o que retarda o crescimento inicial após plantio ou a morte por falta de adaptação ao déficit hídrico, principalmente em regiões onde o período de chuvas é curto e intenso (Davide \& Faria, 2008).

Os resultados obtidos indicam que o uso do hidrogel incorporado ao substrato associado à adubação nitrogenada de cobertura influenciou positivamente $\mathrm{o}$ crescimento e a qualidade das mudas de $H$. ochraceus quando comparados ao tratamento controle. Contudo, as pesquisas sobre o uso do hidrogel associado ou não à adubação na produção de mudas de espécies nativas ainda são incipientes e escassas, ocorrendo, principalmente para espécies de interesse comercial.
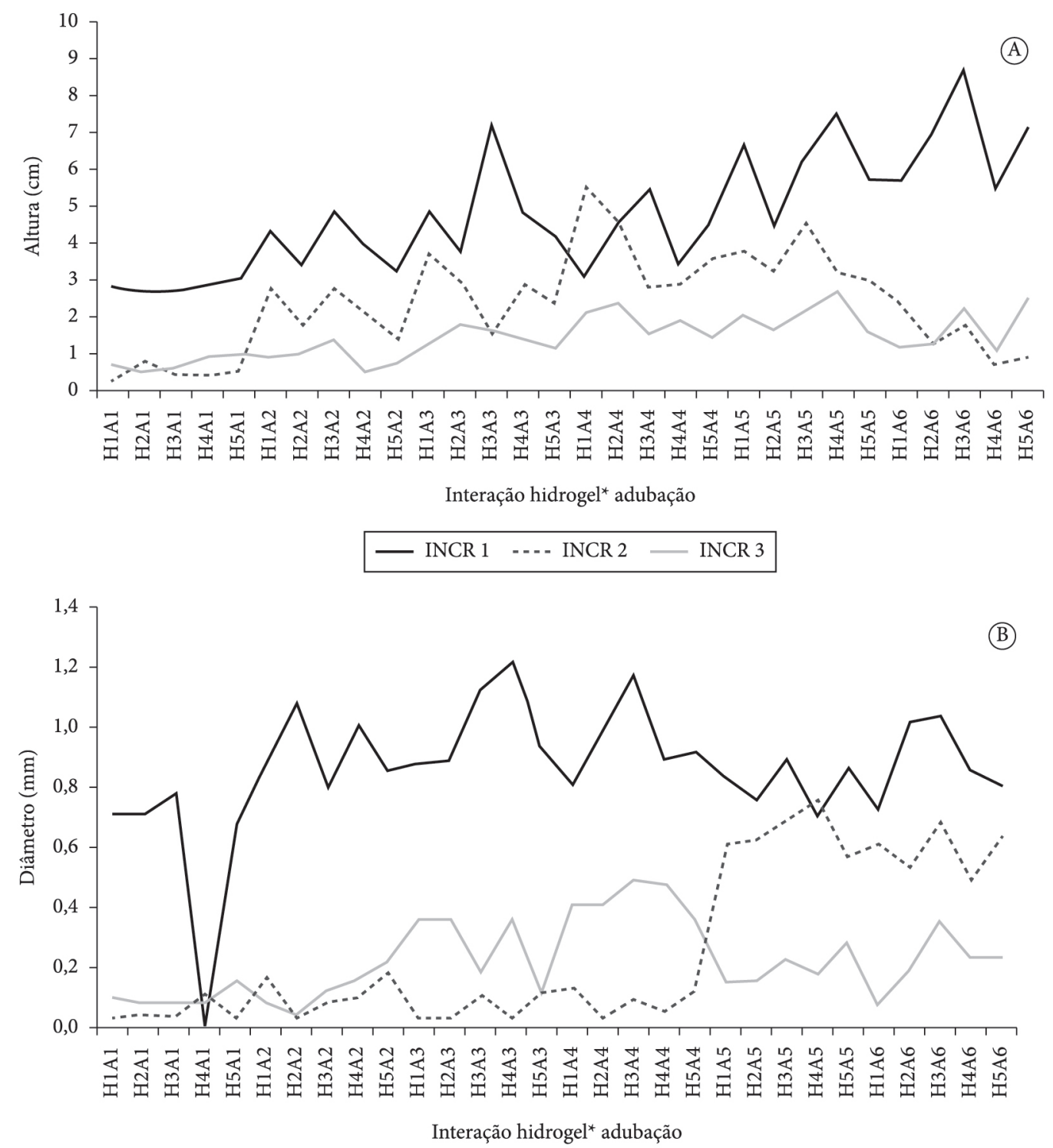

— INCR 1 -.. INCR $2-$ INCR 3

Figura 4. Incremento em altura da parte aérea (A) e diâmetro do coleto (B) em diferentes etapas de avaliação das mudas de Handroanthus ochraceus (Cham.) Mattos submetidas a diferentes doses de hidrogel*adubação em condições de viveiro em Brasília, DF.

Figure 4. Increase in shoot height (A) and stem diameter (B) in different stages of evaluation seedlings Handroanthus ochraceus (Cham.) Mattos, submitted to different doses of hydrogel ${ }^{\star}$ fertilization in greenhouse conditions, Brasília/DF. 
Além disso, em geral as espécies florestais apresentam características distintas de comportamento e respostas, sobretudo quanto às exigências hídricas e nutricionais (Souza et al., 2006). Nesse contexto, ressalta-se a importância e o imediatismo de estudos que produzam informações técnicas e biológicas sobre a efetividade do hidrogel associado à adubação na produção de espécies nativas com potencial silvicultural. Para Handroanthus ochraceus (Cham.) Mattos não há pesquisas relacionadas ao uso da interação hidrogel ${ }^{\star}$ adubação na produção de mudas, o que dificulta a comparação de resultados e, consequentemente, o desenvolvimento de novos estudos com subsídios concisos.

\section{CONCLUSÕES}

A utilização de diferentes doses do polímero hidroabsorvente incorporadas ao substrato associadas à adubação nitrogenada de cobertura influenciou positivamente o crescimento e a qualidade das mudas de Handroanthus ochraceus em condições de casa de vegetação. O maior crescimento em altura da parte aérea e diâmetro do coleto foi observado nos primeiros 80 dias após a implantação do experimento, quando as mudas estavam sob sombreamento de $50 \%$. Para as duas variáveis avaliadas, $\mathrm{H}$ e DC, as dosagens que mais se destacaram em valores de incremento concentraram-se entre 2 a 4 g, tanto para ureia como para hidrogel.

\section{AGRADECIMENTOS}

Ao viveiro Ecotech Tecnologia Ambiental e Consultoria Ltda pela logística, estrutura e auxílio durante o período de estudo.

\section{STATUS DA SUBMISSÃO}

Recebido: 4 mar., 2014

Aceito: 10 out., 2014

\section{AUTOR(ES) PARA CORRESPONDÊNCIA}

\section{Cândida Lahís Mews}

Departamento de Engenharia Florestal, Universidade de Brasília - UNB, CEP 70910900, Brasília, DF, Brasil e-mail: candidamews@gmail.com

\section{REFERENNCIAS}

Azevedo TLF, Bertonha A, Gonçalves ACA, Freitas PSL, Frizzone JA. Níveis de polímero superabsorvente, freqüência de irrigação e crescimento de mudas de café. Acta Scientiarum 2002; 24(5): 1239-1243.

Azevedo TLF. Avaliação da eficiência do polímero agrícola de poliacrilamida no fornecimento de água para o cafeeiro (Coffea arábica L.) cv. Tupi [dissertação]. Maringá: Universidade Estadual de Maringá; 2000

Bernardi MR, Sperotto Junior M, Daniel O, Vitorino ACT. Crescimento de mudas de Corymbia citriodora em função do uso de hidrogel e adubação. Cerne 2012; 18(1): 67-74. http://dx.doi.org/10.1590/S0104-77602012000100009.

Buzetto FA, Bizon JMC, Seixas F. Avaliação de polímero adsorvente à base de acrilamida no fornecimento de água para mudas de Eucalyptus urophylla em pós-plantio. Piracicaba: IPEF; 2002. Circular Técnico n. 195.

Carneiro JGA. Produção e controle de qualidade de mudas florestais. Curitiba: Universidade Federal do Paraná; 1995.

Costa KAP, Oliveira IP, Faquin V. Adubação nitrogenada para pastagem do Gênero Brachiaria em solos de Cerrado. Santo Antônio do Goiás: Embrapa Arroz e Feijão; 2006.

Cruz CAF, Paiva HN, Guerrero CRA. Efeito da adubação nitrogenada na produção de mudas de sete-cascas (Samanea inopinata (Harms) Ducke). Revista Árvore 2006; 30(4): 537-546. http://dx.doi.org/10.1590/S010067622006000400006 .

Davide AC, Faria JMR. Viveiros florestais. In: Davide AC, Silva EAA, editores. Produção de sementes e mudas de espécies florestais. Lavras: Editora UFLA; 2008.

Dickson A, Leaf AL, Hosner JF. Quality appraisal of white spruce and white pine seedling stock in nurseries. Forestry Chronicle 1960; 36(1): 10-13. http://dx.doi.org/10.5558/ tfc36010-1.

Ferrari MP, Shimizu JY. Produção de mudas: cultivo do pinus. Embrapa Florestas; 2005. Sistemas de Produção. [cited 2013 Nov 22]. Available from: http://www.embrapa.br.

Geesing D, Schmidhalter U. Influence of sodium polycrylate on the water-holding capacity of three different soils and effects on growth of wheat. Soil Use and Management 2004; 20(2): 207-209. http://dx.doi.org/10.1111/j.1475-2743.2004. tb00359.x.

Gomes JM, Couto L, Leite HG, Xavier A, Garcia SLR. Parâmetros morfológicos na avaliação da qualidade de mudas de Eucalyptus grandis. Revista Árvore 2002; 26(6): 655-664. http://dx.doi.org/10.1590/S0100-67622002000600002.

Hafle OM, Cruz MCM, Ramos JD, Ramos PS, Santos VA. Produção de mudas de maracujazeiro-doce através da estaquia utilizando polímero hidrorretentor. Revista Brasileira de Ciências Agrárias 2008; 3(3): 232-236. http:// dx.doi.org/10.5039/agraria.v3i3a292.

Kageyama PY, Gandara FB. Recuperação de áreas ciliares. In: Rodrigues R, Leitão Filho HF, editores. Matas ciliares: 
conservação e recuperação. São Paulo: Universidade de São Paulo/FAPESP; 2000.

Lima RLS, Fernandes VLB, Oliveira VH, Hernandez FFF. Crescimento de mudas de cajueiro-anão-precoce 'CCP-76' submetidas à adubação orgânica e mineral. Rev. Bras. Fruticultura 2001; 23(2): 391-395. http://dx.doi. org/10.1590/S0100-29452001000200039.

Lohmann LG. Bignoniaceae. In: Lista de Espécies da Flora do Brasil - REFLORA. 2012. [cited 2013 Nov 10]. Available from: http://floradobrasil.jbrj.gov.br/2012/FB11409.

Lopes PSS, Melo B, Neto FRC, Ramos JD, Carvalho JD. Adubação nitrogenada e substratos no crescimento de mudas de maracujazeiro amarelo em tubetes. Revista da Universidade de Alfenas 1999; 5: 3-8.

Lorenzi H. Árvores brasileiras: manual de identificação e cultivo de plantas arbóreas nativas do Brasil. Nova Odessa: Plantarum; 1992.

Martins CC, Reis EF, Busato C, Pezzopane JEM. Desenvolvimento inicial do cafeeiro conilon (Coffea canephora Pierre) submetido a diferentes turnos de rega e doses de hidroabsorvente. Engenharia da Agricultura 2004; 12(3): 222-228.

Melo ACG, Durigan G, Kawabata M. Crescimento e sobrevivência de espécies arbóreas plantadas em áreas de Cerrado, Assis-SP. In: Vilas Bôas O, Durigan G, editores. Pesquisas em conservação e recuperação ambiental no oeste paulista. São Paulo: Páginas e Letras; 2004.
Nasser RO, Lopes GK, Andrade CT, Teixeira SCS. Correlação entre a capacidade de inchamento e as características estruturais de Polímeros Superabsorventes. In: Anais do 9 Congresso Brasileiro de Polimeros; 2007; Campina Grande. [cited 2013 Feb. 4]. Available from: http://pintassilgo2. ipen.br/biblioteca/cd/cbpol/2007/PDF/291.pdf.

Saad JCC, Lopes JLW, Santos TA. Manejo hídrico em viveiro e uso de hidrogel na sobrevivência pós-plantio de Eucalyptus urograndis em dois solos diferentes. Engenharia Agrícola 2009; 29(3): 404-411.

Sgarbi F, Silveira RVA, Higashi EM, Paula TA, Moreira A, Ribeiro FA. Influência da aplicação de fertilizante de liberação controlada na produção de mudas de um clone de Eucalyptus urophylla. In: Anais do Simpósio sobre Fertilização e Nutrição Florestal; 1999; Piracicaba. Piracicaba: IPEF/ESALQ; 1999. p. 120-125.

Silva MC Jr. 100 Árvores do Cerrado: sentido restrito. Guia de campo. Brasília: Rede de Sementes do Cerrado; 2012.

Sousa GTO, Azevedo GB, Sousa JRL, Mews CL, Souza AM. Incorporação de polímero hidroretentor no substrato de produção de mudas de Anadenanthera peregrina (L.) SPEG. Enciclopédia Biosfera 2013; 9(16): 1270-1278.

Souza PA, Venturin N, Macedo RLG. Adubação Mineral do Ipê-roxo (Tabebuia impetiginosa Martius ex A. P. de Candolle Standley). Ciência Florestal 2006; 16(3): 261-270.

Zar JH. Biostatistical analysis. New Jersey: Prentice Hall; 1999. 